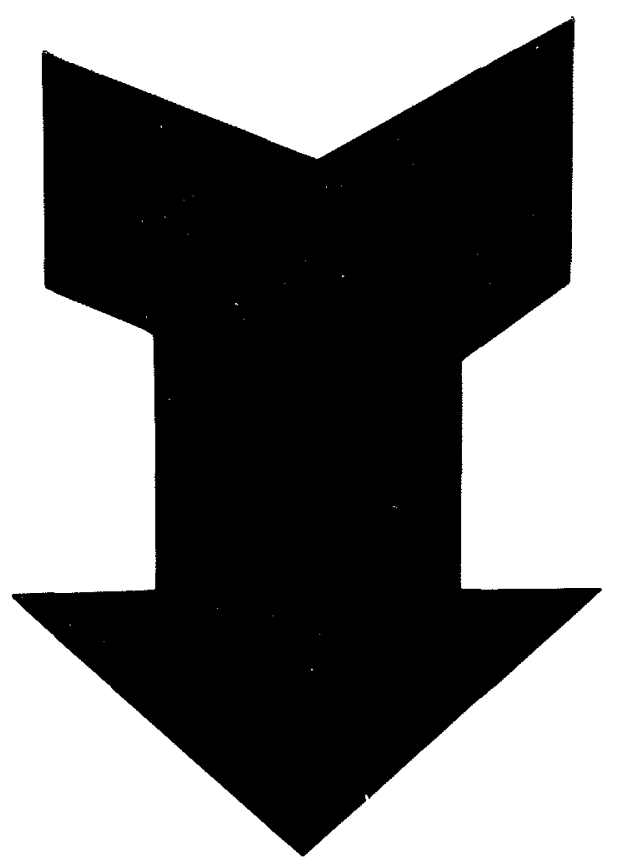

\title{
STATISTIQUES \\ DES HOULES NATURELles MESURÉES PAR \\ LE HOULOGRAPHE AUTONOME TYPE L.N.H.
}

PAR R. BONNEFILLE *,

P. GERMAIN *

ET J.-P. LEPETIT *
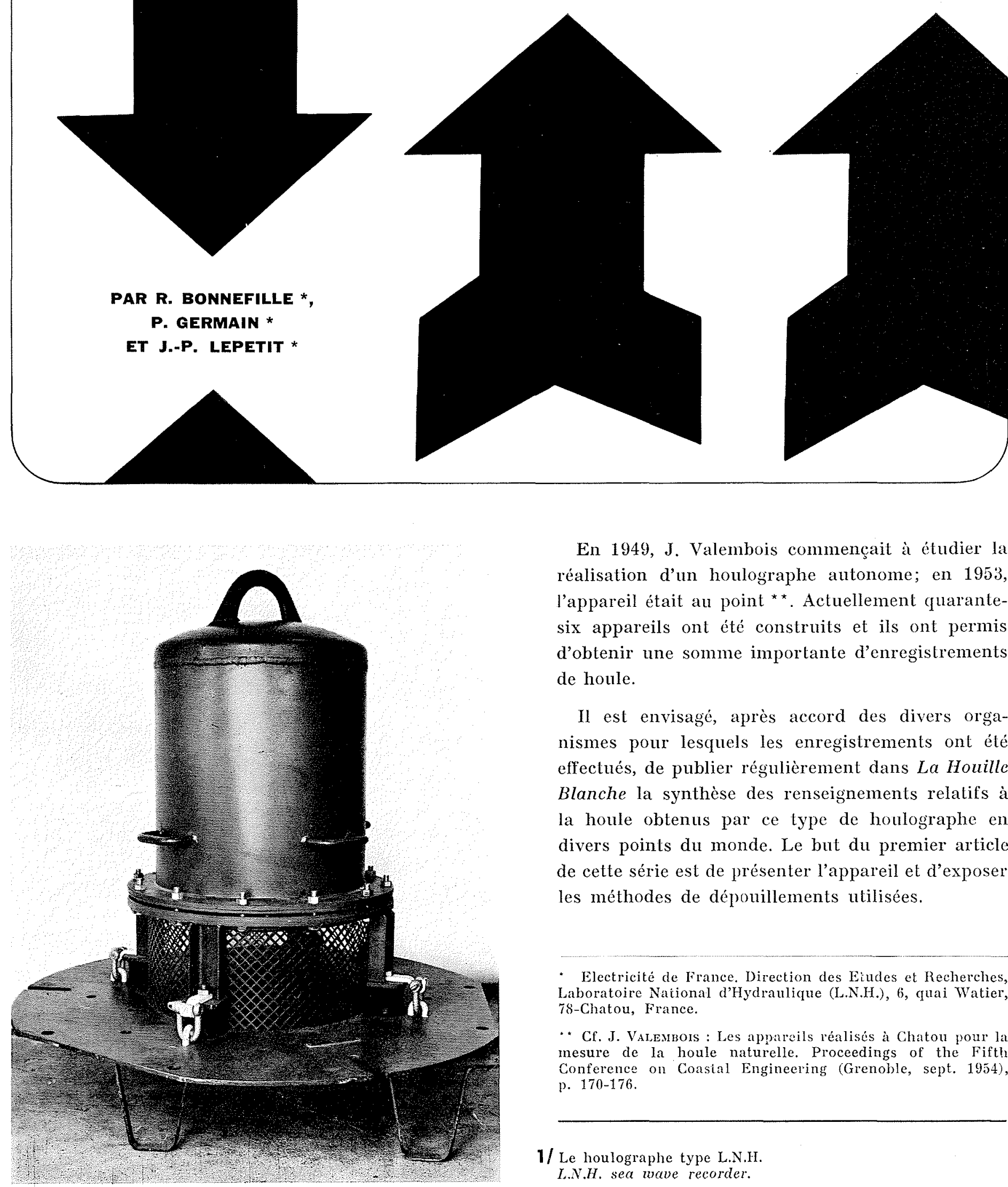

En 1949, J. Valembois commençait à étudier la réalisation d'un houlographe autonome; en 1953, l'appareil était au point **. Actuellement quarantesix appareils ont été construits et ils ont permis d'obtenir une somme importante d'enregistrements de houle.

Il est envisagé, après accord des divers organismes pour lesquels les enregistrements ont été effectués, de publier régulièrement dans La Houille Blanche la synthèse des renseignements relatifs à la houle obtenus par ce type de houlographe en divers points du monde. Le but du premier article de cette série est de présenter l'appareil et d'exposer les méthodes de dépouillements utilisées.

Electricité de France. Direction des Eiudes et Recherches, Laboratoire National d'Hydraulique (L.N.H.), 6, quai Watier, 78 -Chatou, France.

* Cf. J. Valmmors : Les appareils réalisús à Chatou pour la mesure de la houle naturelle. Proceedings of the Fift! Conference on Coastal Engineering (Grenoble, sept. 1954), p. $170-176$.

1/ Le houlographe type L.N.H. I.N.H. sea luave recorder. 


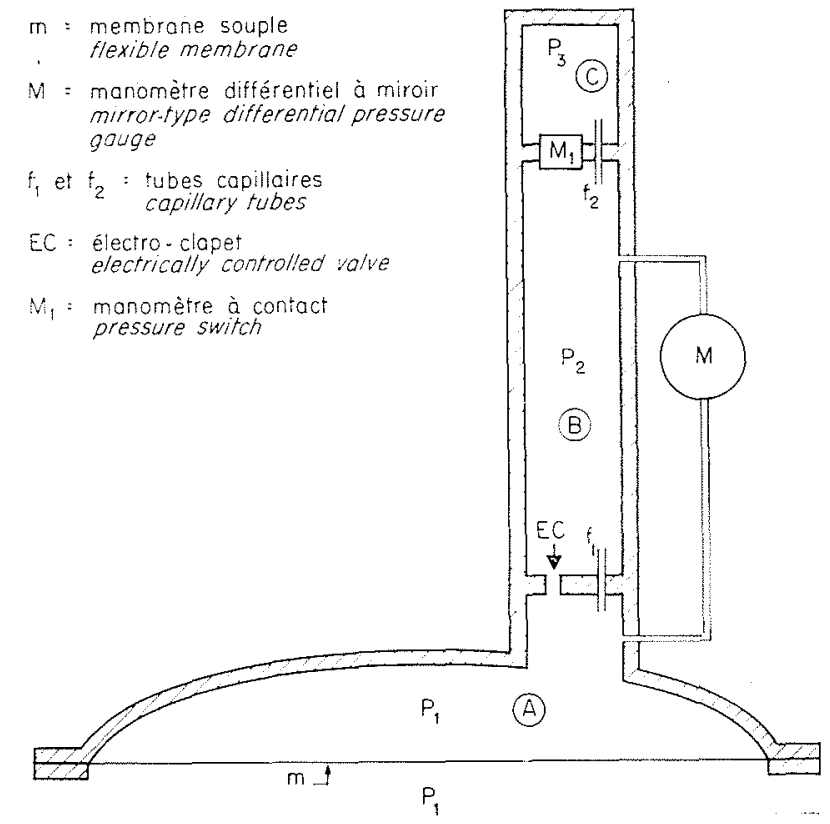

2/

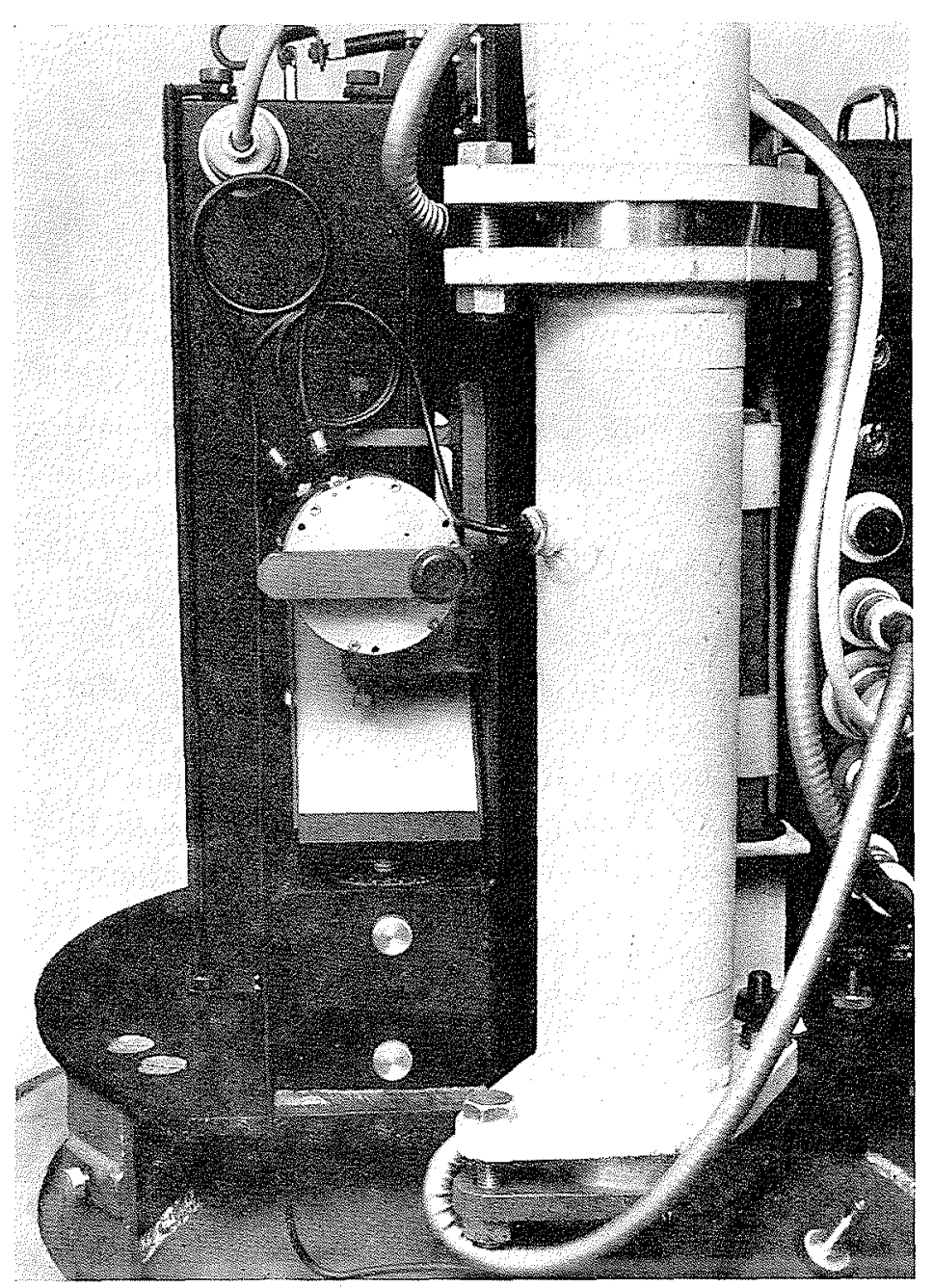

3/ Dispositif de mesure des pressions. Pressure measurement system.

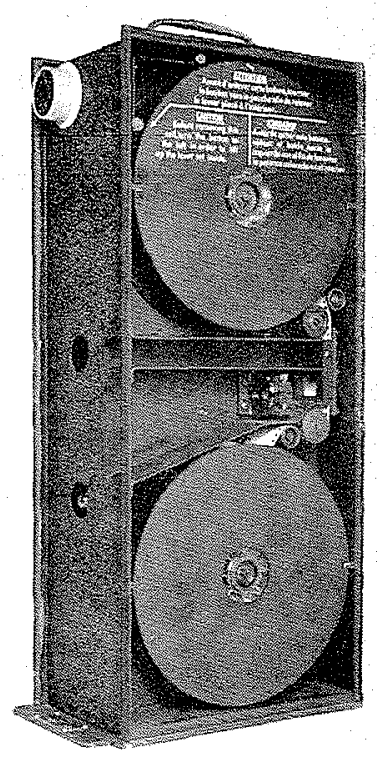

4/ Caméra d'enregistrement.

Recording camera.

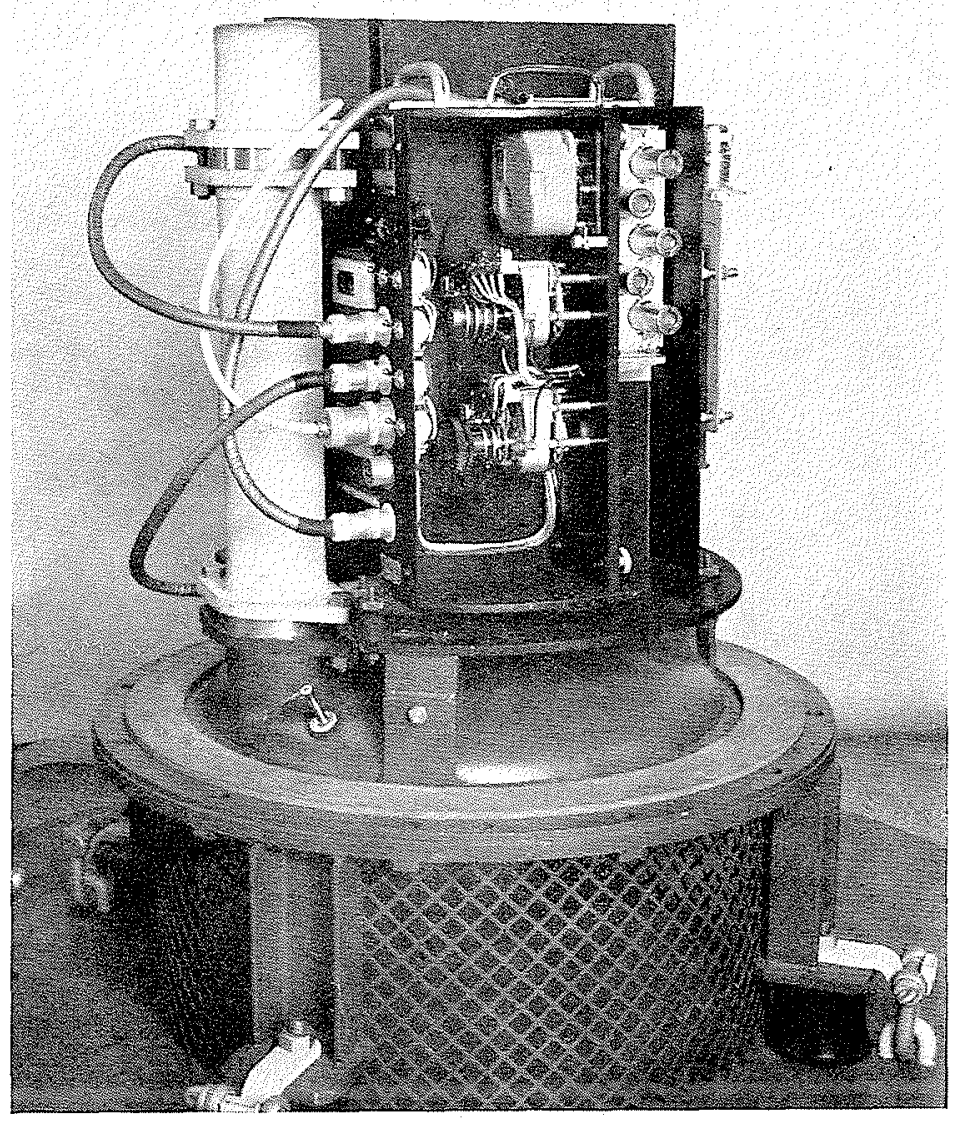

5/Dispositif de commande de l'en'egistrement. Recorder control system. 


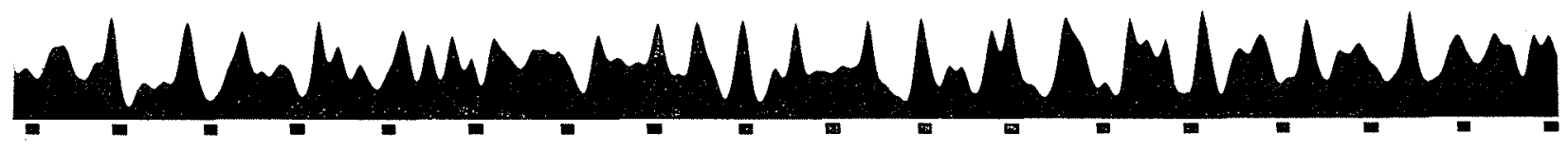

\section{Description de l'appareil et principe de fonctionnement}

L'appareil (fig. 1), posé au fond de la mer, enrezistre les fluctuations de pression dues au passage des vagues. La pression totale $P_{1}$ est transmise par une membrane souple à de l'air contenu dans la chambre (A); cette dernière communique avec la chambre (B) par un tube capillaire $f_{1}$ qui élimine les fluctuations rapides de pression dues à la houle, mais laisse passer les variations lentes de pression (marées, surcotes dues au vent...).

Le manomètre à miroir M (fig. 2 et 3 ), mesure la différence entre la pression instantanée $P_{1}$ dans la chambre (A) et la pression moyenne $P_{2}$ dans la chambre (B). Il n'est donc soumis qu'aux seules fluctuations rapides de pression dues par exemple au passage de la houle. Cette disposition permet d'utiliser un manomètre de grande sensibilité, quelle que soit la profondeur du mouillage.

Les déplacements du miroir du manomètre sont enregistrés sur film de $35 \mathrm{~mm}$, de $120 \mathrm{~m}$ de longueur (fig. 4), pendant 25 minules, toutes les 2 heures, ou toutes les 12 heures, on à chaque pleine mer, on de facon continue, strivant le programme choisi, qui est réglé par un programmateur (fig. 5). Chaque enregistrement comporte (fig. 6) : la photographie d'une horloge quantième donnant l'instant du début de l'enregistrement, et l'inscription sur le bord du film de tops de temps espacés de 30 secondes. La courbe de variation de la pression en fonction du lemps est définie sur le film par la bordure de la plage noire; elle est centrée quelle que soit l'imporlance du marnage.

Le programme d'enregistrement est défini par une horloge commandant par l'intermédiaire de cames et de relais (fig. 5) :

- les enregistrements (éclairage du manomètre à miroir par un spot lumineux, photographie de l'horloge quantième de référence, avance dı film, tops de temps);

l'ouverture entre chaque emregistrement de l'électro-clapet EC, qui court-circuite le tube capillaire $f_{1}$ et permet le monillage rapide de l'appareil entre les enregistrements.

Un dispositif manométrique $\mathrm{M}_{1}$ accélère la cadence des enregistrements à un toutes les 2 heures, lorsque le creux des vagues dépasse une valeur fixée avant le mouillage. Ce dispositif, appelé "contact tempête ", est commandé à partir de la différence entre la pression dans la chambre (C) el la chambre $(\mathrm{B})$

L'autonomie de l'appareil est assurée par une batterie d'accumulateur's; la réserve de marche est de 75,300 on 1200 hemres, suivant la cadence des enregistrements.

Les caractéristiques principales de l'appareil sont :

- poids total : $450 \mathrm{~kg}$;
- hauteur totale: $115 \mathrm{~cm}$;

diamètre de la plaque d'embase : $130 \mathrm{~cm}$.

\section{Activité des houlographes}

Depuis 1952, la fabrication, sous licence E.D.F., est confiée aux Elablissements Saint-Chamond-Granat. Actuellement, vingt-neuf appareils sont en service, soit appartenant au Laboratoire National d'Hydraulique, soit vendus, le L.N.H. assurant le dépouillement des films; 11 appareils sont détériorés ou perdus; le L.N.H. est sans nouvelles de six appareils vendus.

Depuis 1963 , la cadence moyenne de dépouillement des enregistrements de houle s'établit à soixante-cing films par an. Cing cent cinquante films ont été analysés de 1954 à 1966 .

Le tableau ci-après donne la liste des enregistrements de houle disponibles au Laboratoire National d'Hydraulique. On a indiqué en regard le nombre de films correspondant à chaque point d'immersion. Pour traduire en durée d'exploitation, on peut assimiler un film à un mois d'enregistrement.

\section{Principe du dépouillement des films d'enregistrement}

Le but du dépouillement est de caractériser la houle par le plus petit nombre de paramètres. L'enregistrement donne directement les fluctuations de la pression au fond; le problème est de connaitre les oscillations correspondantes de la surface libre.

Soit $p$ la valeur mesurée de la fluctuation de pression, et $h$ la hauteur d'eau correspondante, on admet que le creux de la houle en surface $H$ est donné par la relation

$$
\mathrm{H}=\left(1,25 \operatorname{ch} 2 \pi \frac{d}{\mathrm{I}}\right) h
$$

dans laquelle :

$d$ est la profondeur,

L la longueur d'onde locale;

$$
\mathrm{L}=\left(g \mathrm{~T}^{2} / 2 \pi\right) \text { th }(2 \pi d / \mathrm{L})
$$

T la période de la houle.

Le coefficient 1,25 est un terme correctif déterminé expérimentalement.

La pression est lue en millimètres sur le film. Connaissant l'étalonnage du manomètre, cette valeur est convertible en mètres d'eau. Le creux de la houle est ainsi estimé à 10 à $20 \%$ près.

Il est possible d'analyser l'enregistrement vague par vague, en affectant à chacune la longueur d'onde 
Enregistrements de houle disponibles an Laboratoire National d'Hydranlique à la date du $1^{\text {er juillet } 1967}$

EMPLACEMENT

Nombre DE FILMS

\section{I. - FraNCE :}

Dunkerque : Ports actuel el futur ....... 58

Boulogne : Port ................ 32

Etaples : Baie de la Canche ............ 3

Le Havre : Port .................. 15

Barfleur (Pointe de Gatteville) .......... 3

Cherbourg (Anse de Vauville) .......... 6

Granville (Anse de Hérel) ............. 11

Baie du Mont-Saint-Michel ............. 37

Pointe du Grouin ................... 15

Estuaire de la Rance ................. 7

Cap Fréhel ...................... 2

Brest (Anse de Bertheaume) ............ 7

La Turballe (et Phare du Four) ......... 16

Piriac .......................

Saint-Nazaire (Pointe villes Martin) ...... 3

Chenal extérieur de la Loire ............ 14

La Rochelle ..................... 2

Pointe de Grave (môle d'escale du Verdon). 10

Biscarosse .................... 17

Embouchure de l'Adour ............. 4

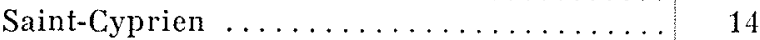

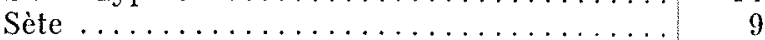

Golfe de Fos :

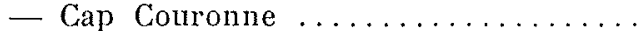

- Bouée la Balancelle ..............

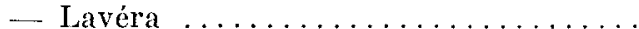

Toulon ......................

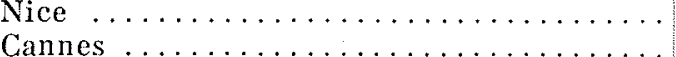

Antibes $\ldots \ldots \ldots \ldots \ldots \ldots \ldots \ldots \ldots \ldots$

II. - BASSIN MÉDITERRANÉEN :

Naples (Italie)

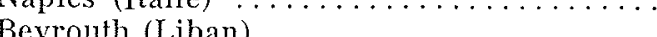

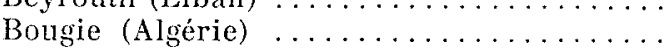

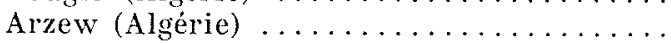

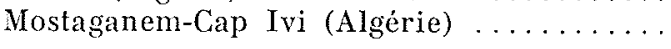

III. - OCÉan ATlantigue :

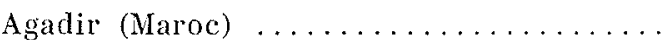

Mansour (Mauritanie) $\ldots \ldots \ldots \ldots \ldots \ldots \ldots$
Rio Nunez (Guinée) . . . . . . . . . . . .

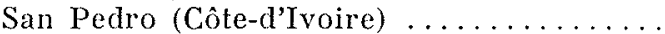

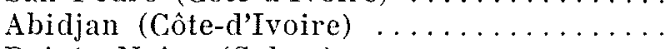

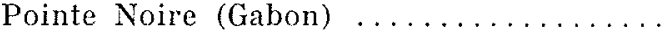

Saint-Pierre-et-Miquelon .............

Comodoro Rivadavia (Argentine)

Estuaire du Gabon ...................

IV. - OCÉAN INDIEN :

Tamatave (Madagascar)

Kuwait (Golfe Persique)

Note. - Nous disposons également de 14 bandes d'enregistrements réalisées au point $\mathrm{k}$ au moyen du «seaborne wave recorder » (Tucker).

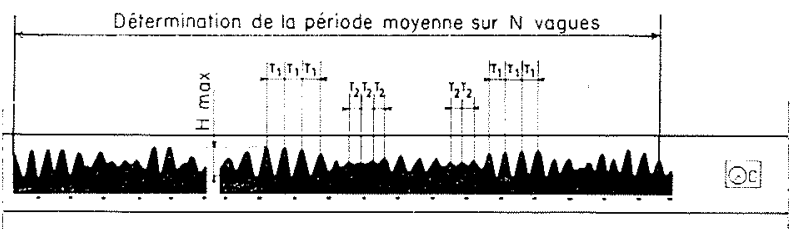

7/ Determination of mean period for ' $N$ ' waves.

correspondant à la période de la vague mesurée sur le film; mais la méthode est longue et pose des problémes d'interprétation difficiles en cas de houle très irrégulière.

La méthode simplifiée utilisée est basée sur les hypothèses suivantes :

- l'enregistrement, qui dure 20 minutes, contient assez d'oscillations pour avoir un sens statistique (200 vagues de 6 secondes ou 100 vagues de 12 secondes);

- le spectre d'énergie du train de houle est assez étroit pour qu'on puisse assimiler le spectre de répartition des creux à un spectre de Rayleigh :

$$
f(\mathrm{H})=\frac{\mathrm{H}}{\mathrm{H}_{0}{ }^{2}} e^{-\mathrm{H}^{2} / 2 \mathrm{H}_{i i^{2}}}
$$

$f(\mathbf{H}) d h$ étant la probabilité d'occurrence du creux $\mathrm{H}$ dans l'intervalle $\mathrm{H}, \mathrm{H}+d \mathrm{H}$, et $\mathrm{H}_{0}$ le creux le plus probable.

Il en découle les relations suivantes, entre les paramètres caractéristiques du train de $\mathrm{N}$ vagues : - creux moyen :

$$
\overline{\mathrm{H}}=1,25 \mathrm{H}_{0}
$$

-.. creux significatif (*) :

$$
\mathrm{H}_{s}=2 \mathrm{H}_{0}=1,6 \overline{\mathrm{H}} \text {; }
$$

- creux maximal :

$$
\mathrm{H}_{\max }=\overline{\mathrm{H}} \sum_{i=1}^{i=1}(-1)^{i+1} \frac{\mathrm{N} !}{(\mathrm{N}-i) ! i ! \sqrt{i}}=\mathrm{H} f(\mathrm{~N})
$$

Pour obtenir $\overline{\mathrm{H}}, \mathrm{H}_{\mathrm{s}}$ ou $\mathrm{H}_{0}$, il suffit donc de connaìtre $\mathrm{H}_{\max }$ et $\mathrm{N}$.

Pratiquement, le dépouillement consiste à compter les vagues de la séquence étudiée, repérer l'oscillation de pression de plus grande amplitude, en déduire $H_{\text {max }}$ en lui affectant, comme période, la période apparente de l'oscillation.

La période moyenne $\bar{T}$ s'obtient facilement en divisant la durée de la séquence par le nombre de vagues; cette valeur très accessible est généralement dépourvue de signification (voir fig. 7).

Dans la plupart des cas, apparaissent deux périodes privilégiées dans les trains de vagues, désignées par ${ }^{2} \mathrm{~T}_{1}$ et $\mathrm{T}_{2}\left(\mathrm{~T}_{1}>\mathrm{T}_{2}\right)$; souvent $\mathrm{T}_{1}$ est associée avec $\mathrm{H}_{\max }$.

(*) On appelle creux significatif $\mathrm{H}_{s}$ ou $\mathrm{H}_{1 / 3}$ d'un train de houle de $N$ vagues, la moyenne des creux des $N / 3$ plus glandes vagues du train de houle considéré. 


\section{Etude statistique des enregistrements de houle}

Les résultats du dépouillement simplifié sont mis sous forme d'un fichier de cartes perforées, à raison d'une carte perforée par séquence, identifiée par la date et l'heure de l'enregistrement. Les cartes «séquence » sont groupées par mouillage, chaque mouillage formant une suite continue d'observations espacées au maximum de 24 heures. Un mouillage est identifié par le lieu et les dates de début et de fin d'immersion du houlographe.

Un programme classe les séquences en creux et périodes de houle. L'intervalle de classement en creux est de $0,2,0,4,0,6$ ou $0,8 \mathrm{~m}$ suivant que l'amplitude la plus grande rencontrée est inférieure à 3 , 6, 9 on $12 \mathrm{~m}$; l'intervalle en période est de 1 seconde. Les séquences correspondant à des amplitudes maximales inférieures à $0,25 \mathrm{~m}$ sont classées dans une rubrique intitulée «calme plat».

Deux classements sont constitués : l'un concerne le creux moyen et la période moyenne, l'autre le creux maximal et la période maximale des trains de vagues.

Les séquences classées dans un même intervalle de creux et de période sont sommées en affectant à chaque séquence un poids égal à l'intervalle de temps sur lequel elle est supposée s'appliquer. Par exemple si $t_{n-1}, t_{n}, t_{n+1}$ sont les époques de trois séquences successives $n-1, n$ et $n+1$, le temps affecté à la séquence $n$ est :

$$
t=\frac{t_{n}-t_{n-1}}{2}+\frac{t_{n+1}-t_{n}}{2}=\frac{t_{n+1}-t_{n-1}}{2}
$$

A la première et à la dernière séquence d'un mouillagge sont affectés les temps :

$$
t=t_{2}-t_{1} \quad \text { et } \quad t=t_{n}-t_{n-1}
$$

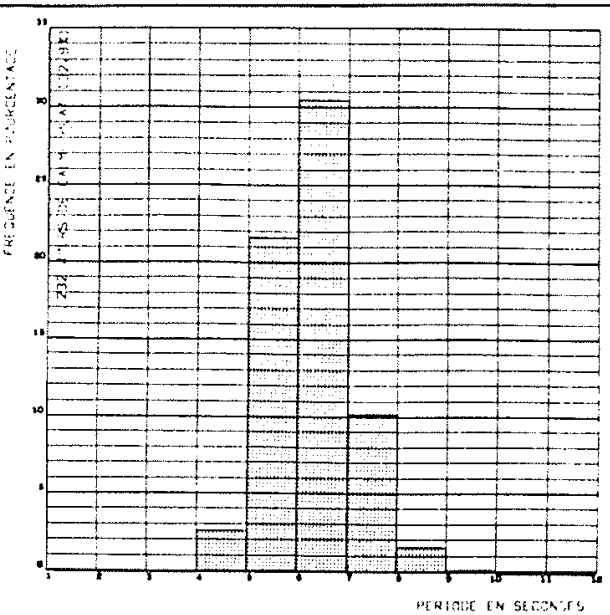

ENREGISTREMENT DE LA HOUE A DUNKEROUE

DU 22-4-60 AU 17-8-66 (706 JOURS 0"OBSERVATION)

HISTOGRAMME DE LA PERIOOE MOYENNE

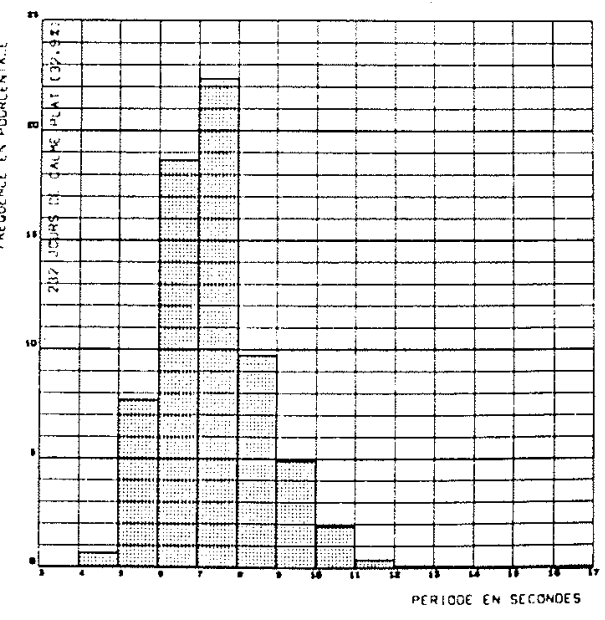

ENREGISTREMENT DE LA HOUE A DUNKEROUE

DU 22-4-60 AU 17-8-66 (706 JOURS D“CBSERVATILN)

histogramme de LA PERIODE maXimale

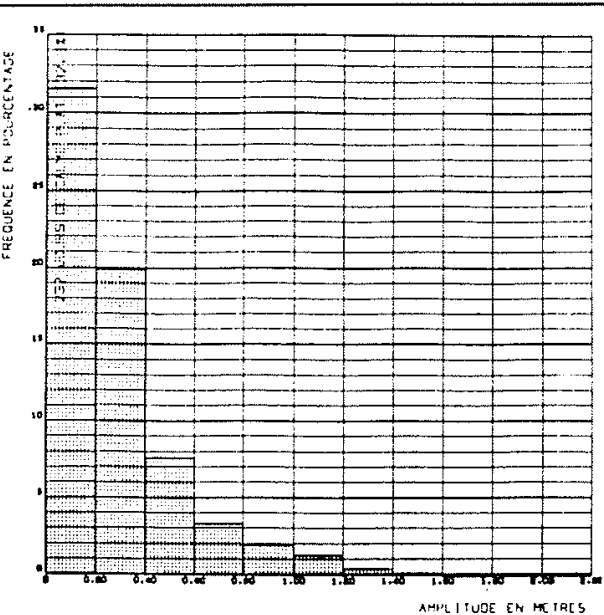

ENREGISTREMENT DE LA HOUE A. DUNKEROUE

OU 22-4-60 AU 17-8-66 (706 JOURS D"OBSERVATION)

HISTOGRAMME DE L"AMPLITUDE MOYENNE

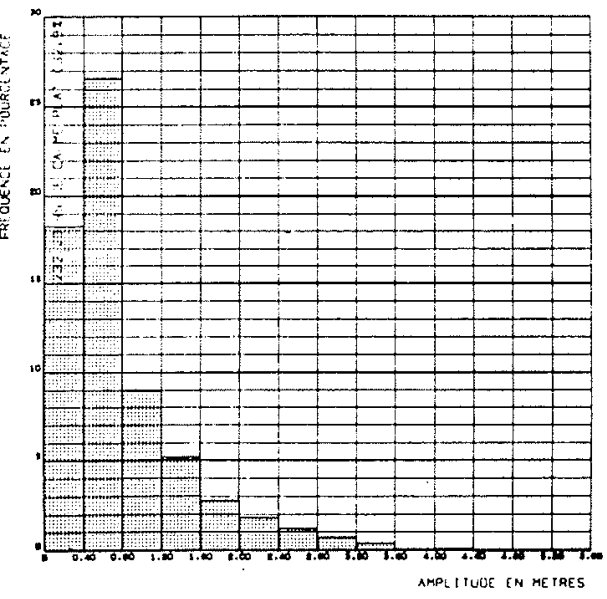

ENREGISTREMENT DE LA HOUL A CUNEROAE

OU 22-4-60 AU 17-8-66 (706 JOURS C"OGSERVATION)

histogramme de L"AMPLITUDE maximale

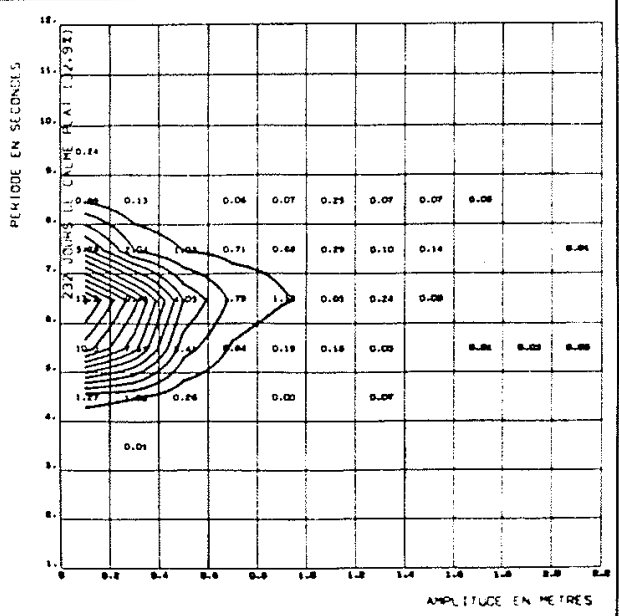

ENREGISTREMENT DE LA HOUE A DNFEROE

D. 22-4-60 AU 17-8-65 (706 JOARS D"COSERVATION) CORRELATION AMPLITUCE MOYENE - PERICOE MOVENE

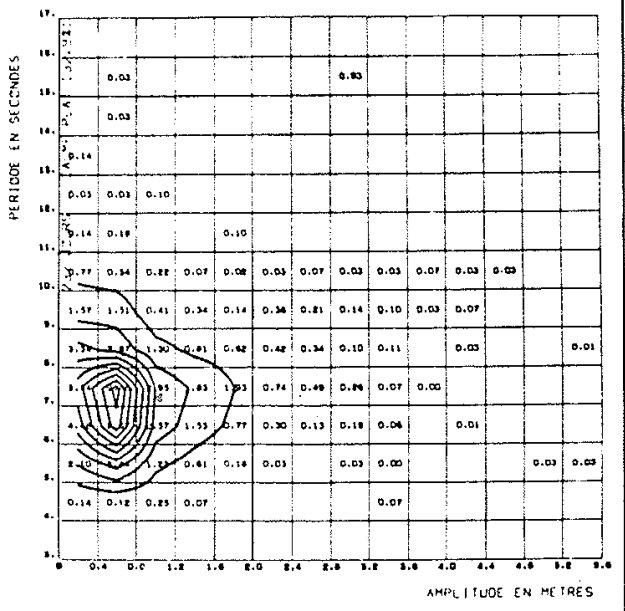

ENREGISTREMENT OE LA HOUE A OUNKRQUE DU 22-4-60 AU 17-8-66 (706 JOURS D"OBSERVATION) CORRELATION AMPLITUOE maXIMALE-PERIODE MAXIMALE

8/ Résultats statistiques du dépouillement de la houle enregistrée ì Dunkerque.

Statistical observed wave data for Dunkirk. 
Le classement el la sommation des séquences sont effectués pour un ensemble de mouillages couvrant tout ou partie de la période analysée. Chaque mouillage est traité indépendamment en ignorant la période entre deux mouillages successifs. Les temps élémentaires des séquences sont cumulés afin d'obtenir le temps total auquel s'appliquent les obser. vations effectuées. Les durées mentionnées dans les lableaux de classement en période el creux sont divisées par ce temps tolal pour obtenir la fréquence en pourcentage.

Ainsi sont établis deux lableaux de corrélation creux-période, donnant dans chaque case la fréquence des observations situées dans des intervalles de creux et de période donnés. Des lignes d'égale fréquence visualisent cette corrélation. La sommation de ces tableaux par ligne, c'est-à-dire par intervalle en période, donne l'histogramme des périodes. La sommation par colonne, c'est-í-dire par intervalle en creux, donne l'histogramme des amplitudes.

L'ensemble des résultats, tableaux de corrélation et histogrammes, est présenté directement sous forme de figures au moyen du traceur de courbes Stromberg Carlson 4020 à écran cathodique. La figure 8 donne $u n$ exemple relatif au port de Dunkerque.

\section{Application de l'étude statistique à l'évaluation de la houle «centenaire » ou « millénaire»}

Le but de l'étude est la connaissance des plus grandes houles « annuelle», « décennale», « centenaire » ou « millénaire » c'est-à-dire les houles de probabilité $1 / 365,1 / 3650,1 / 36500$ on $1 / 365000$.

A partir de l'histogramme de l'amplitude maximale, on peut déterminer, pour chaque valeur $H$ du creux, le pourcentage de temps pendant lequel le creux dépasse cette valeur. Si le nombre d'observations est suffisant et si les observations sont relativement continues, on peut considérer ce pourcentage comme la probabilité de dépasser cette valeur donnée.

Si on connaissait la loi de répartition statistique $\mathrm{P}(\mathrm{H})$, on pourrait en déduire les creux des houles «annuelles», « décennales », etc. J. Larras a proposé la forme mathématique simple suivante qui s'ajuste assez bien aux résultats d'observation el qui permet l'extrapolation jusqu'aux probabilités désirées.

$$
\mathrm{P}(\mathrm{H})=e^{-(\mathrm{H} / \mathrm{A})}
$$

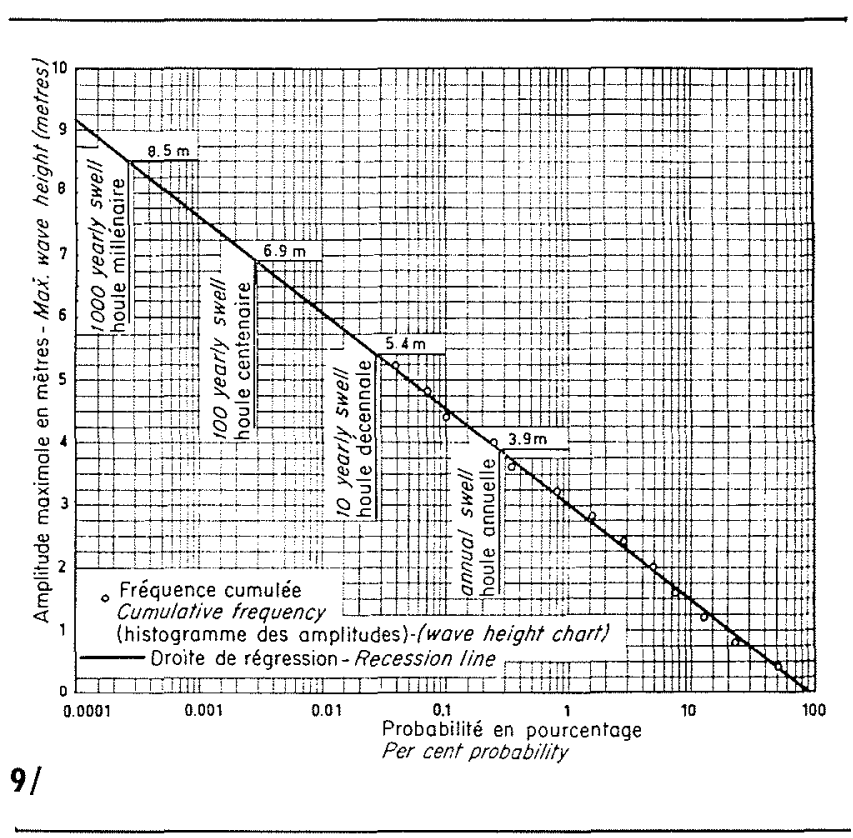

A est un paramètre à déterminer pour chaque point de mesure à partir des observations. Le creux de probabilité $P$ donnée est donc:

$$
\mathrm{H}=-\mathrm{A} \log \mathrm{P}
$$

Les creux des houles «décennale», «centenaire», « millénaire » sont alors liés à celui de la houle annuelle par des relations simples:

- houle décennale. . . . 1,39 $\times$ houle annuelle,

- houle centenaire. . . . 1,78 $\times$ houle annuelle,

- houle millénaire. . . . 2, $2,17 \times$ houle annuelle.

La figure 9 donne un exemple d'application relatif au port de Dunkerque.

\section{Conclusion}

Les enregistrements du houlographe autonome type I.N.H. se prêtent à l'étude statistique de la houle naturelle; une fois l'ensemble du fichier de données constilué sur carles perforées, on peut obtenir très facilement les résultats relatifs à différentes années, saisons ou mois.

En traitant l'ensemble des observations relatives à un lieu donné, on peut en déduire par extrapolation la houle décennale, centenaire, millénaire, à condition que la période d'observation soit suffisamment longue (deux à trois ans) et que la loi des probabilités soit de la forme :

$$
\mathrm{H}=-\mathrm{A} \log \mathrm{P}
$$

